\title{
Racial Landscapes - a pattern-based, zoneless method for analysis and visualization of racial topography
}

\author{
Anna Dmowska ${ }^{\mathrm{a}, \mathrm{b}}$, Tomasz F. Stepinski ${ }^{\mathrm{a}}$, Jakub Nowosad ${ }^{\mathrm{b}}$ \\ ${ }^{a}$ Space Informatics Lab, Department of Geography and GIS, University of Cincinnati, Cincinnati, USA, OH 45221-0131, USA \\ ${ }^{b}$ Institute of Geoecology and Geoinformation, Adam Mickiewicz University, Poznan, Poland
}

\begin{abstract}
Quantifying and effectively communicating the spatio-racial distribution of urban residencies is important for taking the measure of how the multiracial society organizes itself in an urban environment. Most currently used approaches to this problem center around the calculation of segregation metrics; as such, they pertain to only a single pattern's feature and they lack a compelling visualization component. In this paper, we propose a reimagined approach to spatio-racial analysis based on the concept of landscape and landscape analysis. This approach unites quantification and visualization components of the analysis. It also quantifies the entire racial topography, not just segregation. Key novel concepts are the racial landscape (RL) and the exposure matrix. RL is a high-resolution grid in which each cell contains only inhabitants of a single race. The exposure matrix tabulates adjacencies between neighboring cells weighted by the local density of adjacent subpopulations; it provides a concise quantification of the RL pattern. Two information-theoretical metrics, derived from the exposure matrix, quantify diversity, and segregation of the RL. Segregation is quantified from cell adjacencies without the need for subdivision of the region of interest. Thus, the entire region, as well as its arbitrary subregions, are RLs quantified by their diversities and segregations. Coloring cells in $\mathrm{RL}$ according to combinations of their race and local densities provides a natural visualization of racial topography which serves as an "observation" that provides check on numerical metrics. The RL method is described and its application is demonstrated on Cook County, IL. An implementation of the RL method in R package accompanies this paper.
\end{abstract}

Keywords:

Residential segregation, diversity, pattern analysis, landscape, information theory

\section{Introduction}

Population dynamics over the last $\sim 100$ years have been driving ethnoracial changes in cities worldwide (Yizhaq et al., 2004; Bråmå, 2008; Bailey et al., 2012; Finney, 2012). In the U.S., the large changes to urban ethnoracial topography first started to occur in the early twentieth century as black migrants from the South arrived in northern cities, and whites started to depart to the suburbs (Shertzer and Walsh, 2019). In the late twentieth century, significant migration of Hispanics and Asians into the U.S. introduced further changes to urban ethnoracial topography by making it multiracial instead of biracial (Iceland, 2004); this trend continues to this day. Quantifying and communicating etnoracial

\footnotetext{
${ }^{*}$ Corresponding author. Email address: stepintz@uc.edu
}

topography is needed to address multiple urban and social issues (Farrell and Lee, 2011; Maantay and Maroko, 2009; Giordano and Cheever, 2010; Ard, 2016) including access to institutional and economic resources, exposure to crime, pollution, health disparities, and other racial justice issues.

Etnoracial topography (hereafter, for briefness, we will use the term "racial" when it does not lead to confusion) is an overall organization of a spatial point pattern formed by locations of inhabitants' residences and marked by their races (Louf and Barthelemy, 2016). Because the pattern represents a spatio-racial distribution of the multiracial population, quantification of this distribution is tantamount to the quantification of the pattern.

As it happens, the existing literature does not use this pattern paradigm for studying racial topography. Instead, it focuses on the issue of racial segregation which 
is quantified by measuring tendencies of one or more racial groups to be locally over- or under-represented in relation to their city-wide shares. Such focus led to a proliferation of concepts (different flavors of segregations) and metrics associated with them. Segregation has multiple dimensions (Massey and Denton, 1988; Reardon and Firebaugh, 2002; Johnston et al., 2007; Sadahiro and Hong, 2014), varying from as many as five according to Massey and Denton (1988), to as few as two according to Johnston et al. (2007). Also, segregation can be spatial or non-spatial (Wong, 2004; Hong et al., 2014; Yao et al., 2018), as well as global or local (Wong, 2016). Metrics of segregation can be zonebased or surface-based (O'Sullivan and Wong, 2007; Hong et al., 2014), the zone-based metrics are subject to the Modifiable Areal Unit Problem (MAUP) (Openshaw, 1984). Finally, metrics can be measured on a single scale or a multiple scales (Reardon et al., 2008; Lee et al., 2008; Östh et al., 2014; Hennerdal and Nielsen, 2017; Clark et al., 2015; Fowler, 2016; Olteanu et al., 2019). Software packages (Hong et al., 2014; Apparicio et al., 2014) have been developed to help researchers keep track of segregation metrics.

Note that each segregation metric is designed to quantify a single aspect of the spatio-racial pattern. However, many metrics measure the same aspect of the pattern, some quantify aspects of the pattern with little social insight, and some have methodological flaws and ambiguity in interpretation (Hong et al., 2014). This plethora of metrics is rooted in the influential early works by Massey and Denton (1988) and Theil and Finizza (1971). In particular, the very term "segregation metric," inherited from the early literature on the bi-racial, white-black division, could be misleading as it is used as a stand-in for multiple aspects of the racial pattern including diversity and actual segregation. What is referred to as segregation metrics should be instead referred to as metrics of racial topography with the segregation metric being only one of them.

Designs of segregation metrics were influenced by the character of zoned data to which they were originally applied. Later works based on continuous data approximation (Reardon and Sullivan, 2004; O'Sullivan and Wong, 2007; Sadahiro and Hong, 2014) or on gridded data (Östh et al., 2014) continue to use the same metrics even if the different character of the data would allow different metric designs. In addition, segregation metrics are rarely rooted in a theory (for exceptions see Jones et al. (2015) and Louf and Barthelemy (2016)) and therefore they have no interpretation beyond what is inherent to their design. Finally, a significant flaw of existing segregation metrics is that they

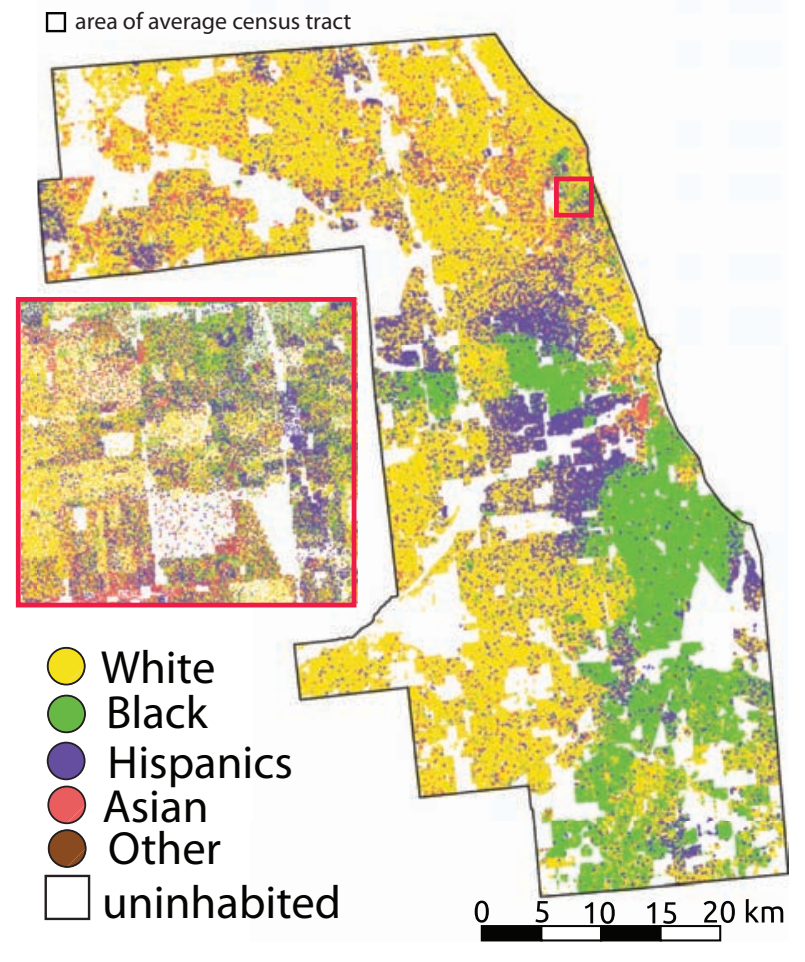

Figure 1: Racial topography of Cook County, IL as depicted by the dot map. Input data to the dot map consists of the high-resolution grid (Dmowska et al., 2017) constructed from 2010 census blocks. The inset shows a small portion of the entire map in greater detail. A typical area of a single census tract is shown by a small square at the top of the figure.

were developed without having a benefit of seeing the actual pattern which they are describing (Stepinski and Dmowska, 2019).

In an attempt to make the racial analysis more coherent and communicative we completely reimagined its methodology. The proposed approach adopts a bird'seye view perspective in which quantification and visualization of racial topography are tightly intertwined a highly accurate analysis and visualization are based on the same data object. Thus, unlike in present methods, the analysis is not performed blind and its results can be verified with "observation." To motivate this approach we start by visualizing, in Fig. 1, the racial topography of the Cook County, IL which includes the urbanized parts of the Chicago metropolitan area. Here, the racial pattern is depicted by the one-person-per-dot map (Roth, 2010; Dmowska and Stepinski, 2019). In such a map each inhabitant of the county is represented by a dot placed at the approximate location of his residence and labeled by a race-specific color; there are $\sim 6$ 
million dots forming the point pattern in Fig. 1. One can grasp the racial topography of Cook County by just looking at Fig. 1.

The racial dot map is an existing technique that provides a very effective visualization of spatio-racial distribution but is not well-suited for quantitative analysis because of its spatial point pattern design. The aim of the proposed method is to provide a theory-rooted quantification of spatio-racial patterns that naturally leads to a visualization which is as effective as the dot map. The method starts with the construction of a regular, highresolution racial grid that is well-suited for quantitative, theory-rooted analysis (see section 2.1 and 2.2). Treating this grid as a "image" raster, where each cell is assigned a color on the basis of its race and population density, provides a natural visualization. Note that this is a racial grid different from previously introduced diversity/dominant race grids (Dmowska et al., 2017). As a data object, the racial grid structurally resembles a concept of "landscape" used in the domain of landscape ecology (Gergel and Turner, 2017), therefore we will call it the racial landscape (RL), and will refer to the entire method as the racial landscape method (RL method).

Whereas the census divides an urban area into zones (for example, census tracts) along arbitrarily drawn boundaries, the RL has no divisions, but its visualization clearly shows a mosaic of many large and small patches formed by adjacent grid cells having the same race labels. To quantify the RL we use the Information Theory-based approach which recently has been developed (Nowosad and Stepinski, 2019) in the context of landscape ecology. In this approach, the racial pattern (in the entire region or in any arbitrary subregion) is quantified by two information-theoretical metrics (see section 2.3), one describes racial diversity and another racial segregation. These two metrics are not only the most important features of the spatio-racial pattern from a demographic point of view, but they also have been shown (Nowosad and Stepinski, 2018) to be weakly correlated and, together, they explain $\sim 70 \%$ of the pattern's variability.

The goal of this methodological paper is to introduce and describe the RL method of analyzing racial topography. After the key concepts of the RL method are introduced in section 2, we demonstrate in section 3 the working of the method using the Cook County, IL as an example. Discussion and conclusions follow in section 4.

\section{Method description}

Input data for the construction of RL is the racial composition of the population at the U.S. Census Bureau block-level aggregation. We consider five subpopulations: White, Black, Asian (which include Hawaiian/Pacific Islanders), Hispanics, and others (which include American Indians. The Census tabulates population according to race, and separately, according to ethnicity. We use a division that combines these two tabulations, the "Hispanics" category groups inhabitants of Hispanic ethnicity, remaining categories divides inhabitants of non-Hispanic ethnicity on the basis of their race. Blocks are rasterized to a grid of $30 \mathrm{~m} \times 30 \mathrm{~m}$ cells. The next step is to assign racial composition (percentages of the total population belonging to racial subpopulations) to each cell individually. There are two possibilities to proceed: (1) cells inherit subpopulation percentages from a block to which they belong, and (2) cells are assigned racial composition using the SocScape gridded data (Dmowska et al., 2017). SocScape (http://sil.uc.edu/webapps/socscape_usa) provides dasymetrically modeled gridded population and race data for the entire conterminous U.S. in 1990, 2000, and 2010. SocScape data provides a preferable input because it is already gridded and it does not place people in cells located in uninhabited parts of blocks. In this paper, we use SocScape data.

\subsection{Constructing racial landscape}

Racial composition at each cell is translated into probabilities of drawing a person of a specific race from a cell. Thus, the race label of a cell is not deterministic; instead, it is a random variable. To obtain a stochastic realization of RL we use cell's race probabilities and a random number generator to randomly assign a specific race label to each cell (the Monte Carlo procedure). The example of a single realization of RL is shown in Fig. 2B.

Comparing the RL shown in Fig. 2B with the dot map of the same area shown in Fig. 2A, we notice that RL provides a skewed visualization of the racial pattern because it does not take into consideration spatial variability of the population density. To obtain an accurate depiction of the racial distribution the values of the RL must be modified to reflect race and a local population density. We calculate a local density of a given subpopulation at each cell taking care to preserve the total number of people at each sub-population at all scales.

A density-modified RL is shown in Fig. 2C. It provides an accurate visualization of the racial topography, arguably even better than the dot map which becomes 


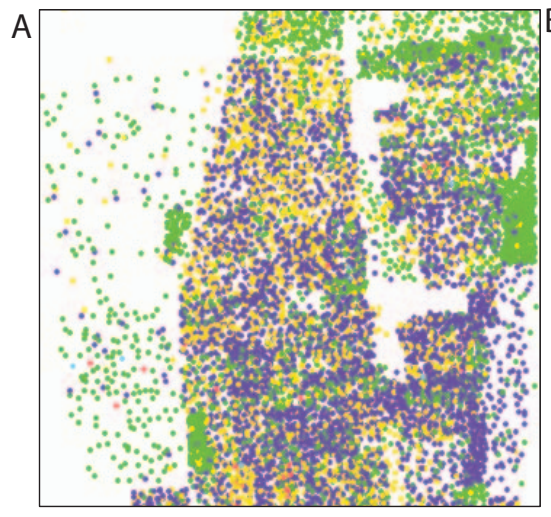

White

Black

Hispanics

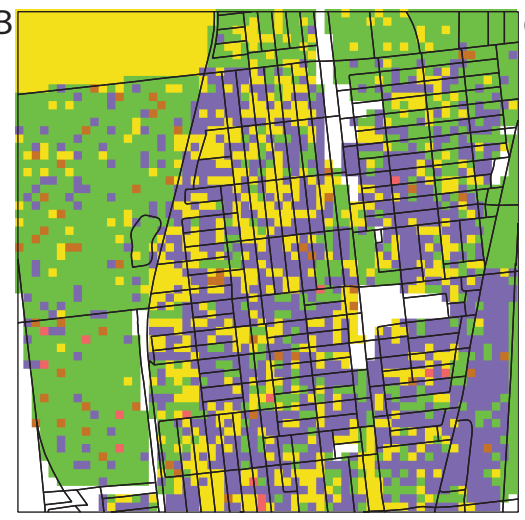

Asian

Others

uninhabited

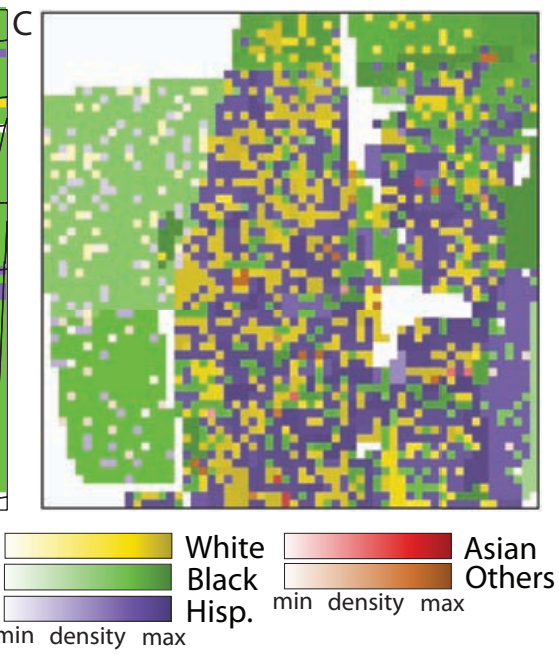

Figure 2: Visualization of racial topography in a $1.8 \mathrm{~km} \times 1.8 \mathrm{~km}$ region in Chicago, IL. (A) The racial dot map. (B) A stochastic realization of racial landscape with superimposed boundaries of 264 constituent census blocks. (C) Density-modified realization of the racial landscape.

saturated (individual dots are not recognizable) in regions with high population density. Note that whereas the original RL is a categorical grid (each cell is labeled by one of few races depicted by few colors), the density modified RL is a numerical grid (each cell has a different color in order to depict both race and density).

\subsection{Co-occurrence and exposure matrices}

We quantify the RL pattern by the co-occurrence matrix (Haralick et al., 1973) - a 2D histogram of adjacencies between cells in the RL grid. The adjacency between cells is defined by the rook's rule (4connectivity); the region's internal cells have four adjacencies (north, east, south, and west) whereas cells on the boundary of the region have fewer adjacencies. Cells corresponding to uninhabited areas are considered as no data; adjacencies with such cells do not contribute to the co-occurrence matrix. An adjacency can also be thought of as an ordered pair $(x, y)$, where $x$ is the race label of the focus cell and $y$ is the race label of an adjacent cell. The labels are from the set $\left\{r_{1}, \ldots, r_{K}\right\}$ of $K$ races present in the pattern; $K=5$ for data used in this paper. The co-occurrence matrix has the size $K \times K$ and is symmetric; it can be calculated for any region regardless of its size and shape.

The co-occurrence matrix (CCM), which is a tabulation of cells' adjacencies, is a compact quantification of an areal pattern (like the one shown in Fig. 2B). We modify the CCM algorithm to obtain an exposure matrix (EM) which compactly quantifies an amount of exposure between different races, and thus the structure of racial topography. The EM is calculated in the

\begin{tabular}{|c|c|c|c|c|c|c|c|}
\hline$x y$ & W & $B$ & $A$ & $\mathrm{H}$ & 0 & $\sum_{\mathrm{y}}$ & $H\left(y \mid x_{i}\right)$ \\
\hline 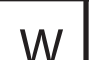 & 3206 & 1810 & 50 & 3910 & 91 & 9067 & 1.626 \\
\hline & & & & & & & \\
\hline & 1810 & 7301 & 62 & 3172 & 123 & 12468 & 1.462 \\
\hline & 50 & 62 & 10 & 41 & 0 & 163 & 1.799 \\
\hline & 3910 & 3172 & 41 & 9311 & 172 & 16606 & 1.505 \\
\hline & 91 & 123 & 0 & 172 & 12 & 398 & 1.686 \\
\hline$y$ & 9067 & 2468 & 163 & 16606 & 398 & $\begin{array}{l}H(x)= \\
H(x)=\end{array}$ & \\
\hline $\mathrm{X}$ & 目 & $\square$ & 目 & & & $\begin{array}{l}\mathrm{H}(\mathrm{y})= \\
1.642\end{array}$ & \\
\hline$\left.x \mid y_{j}\right)$ & 1.626 & 1.462 & 1.799 & 1.505 & 1.686 & & $\begin{array}{l}H(x \mid y)= \\
H(y \mid x)= \\
1.523\end{array}$ \\
\hline
\end{tabular}

Figure 3: Exposure matrix for the RL shown in Fig. 2. The EM is the part of the figure within a heavy line square. EM entries are rounded to the nearest integer. Capital letters indicate race category: W-whites, B-Blacks, A- Asians, H-Hispanics, O-others. Types of exposure are indicated by pairs of colored cells; for example, the exposure of whites to blacks (and vice-a-versa) is 1810. The seventh column (raw) lists total exposures of races present in the RL; for example, the total exposure of whites to other races is 9067 of which 3206 is a self-exposure. Color-coding of races is the same as in Figs. 1 and 2.

same way as the CCM but each adjacency contributes a location-specific value to the matrix instead of the constant value of 1 . The contributed value is an average of population densities in the two adjacent cells. 
Table 1: Information Theory metrics

\begin{tabular}{lll}
\hline name & formula & measures \\
\hline \hline joint entropy & $H(x, y)=-\sum_{i=1}^{K} \sum_{j=1}^{K} p\left(x=r_{i}, y=r_{j}\right) \log _{2} p\left(x=r_{i}, y=r_{j}\right)$ & racial pattern complexity \\
conditional entropy & $H(y \mid x)=-\sum_{i=1}^{K} \sum_{j=1}^{K} p\left(x=r_{i}, y=r_{j}\right) \log _{2} p\left(y=r_{i} \mid x=r_{j}\right)$ & diversity of race adjacencies \\
marginal entropy & $H(y)=-\sum_{j=1}^{K} p\left(y=r_{j}\right) \log _{2} p\left(y=r_{j}\right)$ & diversity of races \\
mutual information & $I(y, x)=H(y)-H(y \mid x)$ & racial segregation \\
\hline
\end{tabular}

$p\left(x=r_{i}, y=r_{j}\right)$ - a probability of the focus cell having a race $r_{i}$ and an adjacent cell having a race $r_{j}, p\left(y=r_{i} \mid x=r_{j}-\right.$ a probability of the adjacent cell havening a race $r_{i}$ providing that the focus cell has a race $r_{j}, p\left(y=r_{j}\right)=\sum_{i} p\left(x=r_{i}, y=r_{j}\right)$ a probability that an adjacent cell has a race $r_{j}$. In the IT the logarithm with base 2 is used so the information is measured in bits. For derivation of these metrics in the context of spatial patterns see Nowosad and Stepinski (2019).

Fig. 3 depicts the EM for the region shown in Fig. 2 (it is calculated using adjacencies as depicted in Fig. 2B and densities as depicted in Fig. 2C). Diagonal entries in the EM measure self-exposure for the five subpopulations. Large values of diagonal entries relative to values of off-diagonal entries indicate significant aggregation of same-race inhabitants. Off-diagonal entries in the EM measure inter-race exposure between pairs of sub-populations. Relatively large values of off-diagonal entries indicate disaggregation of same-race inhabitants.

\subsection{Information Theory metrics}

Although the EM is a compact description of an RL, further compression of information in EM is needed for the lucid quantification of racial topography. Nowosad and Stepinski (2019) recently proposed to quantify patterns using the information theory (Shannon, 1948). The Information Theory (IT) uses the concept of entropy to quantify the amount of information associated with a given probability distribution function. Normalizing the EM (dividing it by the sum of all entries) yields a joint probability distribution function $p(x, y)$, where $p\left(x_{0}, y_{0}\right)$ is a probability of drawing from the RL an adjacent pair of cells labeled by races $x_{0}$ and $y_{0}$, respectively.

The entropy $H[p(z)]=H(z)=-\sum p(z) \log _{2} p(z)$ is a number that measures the information the distribution $p(z)$ provides about the variable $z$. Here, $z$ indicates either one or two-dimensional variable, and the summation is over all unique values of $z$. Entropy is a property of distribution, thus, in our case, a property of the RL. We explain the idea behind the formula for the entropy following the description in Frank (2009). The value $-\log _{2} p(z)$ increases as the probability $p(z)$ becomes smaller. Thus, it measures the surprise in observing a particular value of $z$; rare events are more surprising.
Greater surprise provides more information, if we are not surprised by observation we gain little information (we knew a priori what we would get). Thus entropy is an average of surprise (information) over all possible values of $y$. A useful interpretation of entropy is that it is an expected number of optimally asked yes/no questions a person familiar with the probability distribution needs to ask to determine the value of randomly drawn $z$ variable. The distribution contains more information if more questions need to be asked.

Table 1 provides formulas for entropies of four different probability distribution functions associated with the RL. Nowosad and Stepinski (2019) demonstrated that the first three metrics are highly correlated with each other but weakly correlated with $I(y, x)$. Thus, we use only $H(x)$ and $I(y, x)$ to quantify the racial landscape. $H(x)$, has a theoretical range between 0 and $\log _{2} K$, it measures the diversity of racial composition; it is the same metric as is used in current methods where it is customarily denoted by the symbol $E$.

$I(y, x)$, called the mutual information, is a reduction of uncertainty as to the race of the cell if one knows the race of its neighbor; it has a theoretical range between 0 and $H(x)$. For racially homogeneous site $I(y, x)=0$ because there is no uncertainty associated with the homogeneous site, and thus nothing to reduce. In a site consisting of equally abundant subpopulations randomly distributed throughout its extent $I(y, x)=0$ because knowing the race of a cell does not provide any extra information about the race of an adjacent cell. In a site consisting of equally abundant subpopulations with each subpopulation concentrated in its own enclave $I(y, x)$ achieves its maximum value because knowing a race of a cell determines the race of an adjacent cell except for cells located on boundaries between racial enclaves. Clearly, $I(y, x)$ measures racial segregation. 


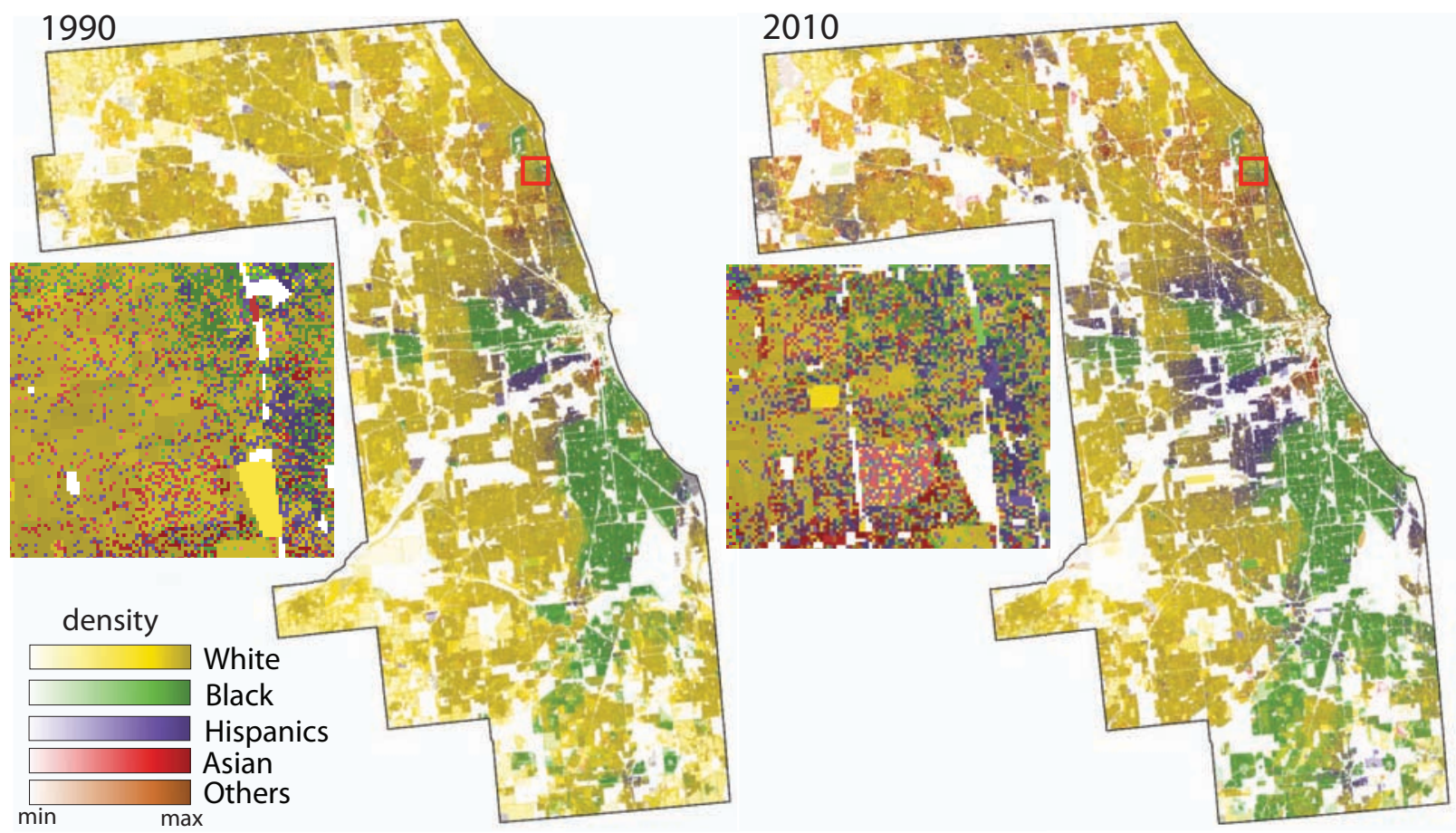

Figure 4: Density-modified racial landscapes for Cook County, IL in 1990 (left) and 2010 (right). Insets show a magnification of the small portion of the county indicated by a red rectangle.

Note that $I(y, x)$ is a conceptually different measure of segregation than the Theil's information theory index $\mathcal{H}$ (Reardon and Firebaugh, 2002; Reardon and Sullivan, 2004). It can be calculated for the entire region or an arbitrary subregion because it utilizes only cells' adjacencies instead of internal zones like the $\mathcal{H}$ does.

In a site shown in Fig.2 the maximum possible diversity would be $H(x)=2.32$ if the site would have an equal number of people in all five races. An actual racial composition of the site is $\mathrm{W}-23 \%, \mathrm{~B}-33 \%$, $\mathrm{A}$ $-0.4 \%, \mathrm{H}-42.6 \%, \mathrm{O}-1 \%$, resulting in $H(x)=1.64$. Correct visual inspection of this site does not indicate segregation. This observation is confirmed by the low value of $I(y, x)=0.12$. Note that an incorrect inspection of Fig. 2C could focus on the existence of large, light green patches, however, these are low-density regions that do not contribute much to the value of populationbased segregation measure.

\subsection{Stochastic character of $R L$}

In sections 2.1 to 2.3 we explained how to visualize and quantify a single stochastic realization of RL. Multiple draws yield a series of RL realizations with slightly different patterns. The pattern uncertainty occurs only at the sub-block scale and only if there is a significant sub- block racial diversity. For example, for the site shown in Fig. 2, which covers over 260 blocks, differences between patterns of different realizations of RL are negligible. As a result, a single realization-based visualization of RL and IT metrics are sufficiently accurate. For increased accuracy, we quantify racial topography by calculating IT metrics as ensemble averages from multiple realizations of RL.

\section{Application to the Cook County, IL}

Fig. 4 shows density-modified RLs for the Cook County in 1990 and 2010. Each RL is a single stochastic realization (see section 2.4). Small-scale racial topography may not be visible in a raster rendered to the size and resolution of the journal page. Insets in Fig. 4 show the detailed racial pattern in a small portion of the county. The supplement to this paper contains GeoTIFF files of RLs depicted in Fig.4. Each RL is formed by five (one for each race) GeoTIFFs, so the spatial distribution of each race can be seen separately if needed. Note that the RL is constructed so cells representing different races form a mutually exclusive and exhaustive set, thus there is no overlapping between spatial distributions of different races. The 2010 RL in Fig.4 visually resem- 
Table 2: Values of pattern IT metrics for regions of different scales

\begin{tabular}{l|lll|lll}
\hline & \multicolumn{3}{|c|}{1990} & \multicolumn{3}{c}{2010} \\
\hline scale & $\#$ & $\langle H(x)\rangle$ & $\langle I(y, x)\rangle$ & $\#$ & $\langle H(x)\rangle$ & $\langle I(y, x)\rangle$ \\
\hline $1.8 \mathrm{~km}$ & 2439 & $0.65(2)$ & $0.044(4)$ & 2226 & $1.05(2)$ & $0.042(5)$ \\
$3.6 \mathrm{~km}$ & 623 & $0.740(8)$ & $0.086(3)$ & 566 & $1.135(4)$ & $0.076(4)$ \\
$7.2 \mathrm{~km}$ & 157 & $0.874(4)$ & $0.163(2)$ & 138 & $1.293(3)$ & $0.144(3)$ \\
$14.4 \mathrm{~km}$ & 36 & $1.015(2)$ & $0.253(2)$ & 32 & $1.420(2)$ & $0.234(2)$ \\
county & 1 & $1.5335(9)$ & $0.5509(8)$ & 1 & $1.8422(8)$ & $0.4685(8)$
\end{tabular}

Ensemble averages and standard deviations of IT metrics are listed in one compact notation. For example, for the scale $14.4 \mathrm{~km}$ in 1990 , the value of $\langle H(x)\rangle$ is 1.015429 and the standard deviation is 0.0021695 , resulting in Table 2 entry $1.015(2)$. The value of a metric is rounded to the digit corresponding to the first position of the value of standard deviation. The value of standard deviation is rounded to one significant digit.

bles the dot map shown in Fig. 1. However, whereas the dot map is a collection of over 6 million irregularly placed and arbitrarily sized dots, the RL is a regular grid with cells having real physical dimensions.

For the Cook County dataset (in 1990 and, separately, in 2010) we have calculated 100 stochastic realizations. We calculated the values of $H(x)$ and $I(y, x)$ for each realization. The ensemble-averaged values of $\langle H(x)\rangle$ and $\langle I(y, x)\rangle$ and their standard deviations are given in the last row of Table 2. Note that the values of standard deviations are very small in comparison to the values of averages. This means that at the scale of the entire county differences in patterns of individual realizations are very small.

According to Table 2 the racial diversity of Cook County increased by $\approx 0.3$ during the $1990-2010$ period. The IT interpretation of this difference is as follows: to learn the race of random Cook County inhabitant in 2010 we need to ask, on average, 0.3 more optimal questions than in 1990. Still, we only need to ask 1.84 questions, whereas we would need to ask $\log _{2} 5 \approx 2.32$ questions if all five racial subpopulations were equally abundant. The value of $\langle I(y, x)\rangle$ has decreased by $\approx 0.08$ during the 1990-2010 period indicating a small reduction of racial segregation. For an illustration of what $\langle I(y, x)\rangle \approx 0.08$ means in terms of change in spatioracial pattern see Stepinski and Dmowska (2019).

The RL method is ideally suited for multiscale analysis. Note that in the RL method the term scale refers to the measurement scale, not the geographical scale. Consider a square site having the side length $L$ ( $L$-site) randomly placed in Cook County. The racial topography of this site is calculated the same way as for the entire county except using only a portion of the RL restricted to the extent of the $L$-site. The racial topography of the
$L$-site depends on its location within a county. A typical racial pattern on the spatial scale $L$ in the Cook County is quantified by average values of $H(x)$ and $I(y, x)$ calculated over a set of $L$-sites covering (with an overlap) the entire county. The first four rows in Table 2 shows the results for values of $L$ equal to $1.8 \mathrm{~km}, 3.6 \mathrm{~km}, 7.2 \mathrm{~km}$, and $14.4 \mathrm{~km}$. The second column in Table 2 gives the number of overlapping $L$-sites at each scale, note that the numbers of $L$-sites in 1990 and 2010 are slightly different due to different extents of uninhabited areas.

In a given year, values of both $\langle H(x)\rangle$ and $\langle I(y, x)\rangle$ decrease with decreasing spatial scale - the racial pattern on the smaller scale is more homogeneous and less segregated than on the larger scale meaning that the racial diversity and segregation decrease with decreasing scale. Temporally, during the 1990-2010 period, values of $\langle H(x)\rangle$ (racial diversity) increased on all scales, and the values of and $\langle I(y, x)\rangle$ (racial segregation) decreased on all scales.

The values in the first and the last rows in Table 2 can be compared to the results in the recent paper by Stepinski and Dmowska (2019) who also utilized the ITbased metrics to assess racial diversity and segregation but using the zoned data (county subdivided into tracts and tracts subdivided into blocks). For the Cook County their method yields $H(R)=1.55$ and $I(R, S)=0.81$ in 1990 and $H(R)=1.86$ and $I(R, S)=0.77$ in 2010 . For tracts in the Cook County, their method yields $\langle H(R)\rangle=$ 0.74 and $\langle I(R, S)\rangle=0.11$ in 1990 and $\langle H(R)\rangle=1.05$ and $\langle I(R, S)\rangle=0.19$ in 2010 , where averaging is over all tracts in the county. Here $R$ stands for race variable and $S$ stands for place variable (which tract in the county or which block in a tract).

Despite the fact that Stepinski and Dmowska (2019) used different data representations and different vari- 


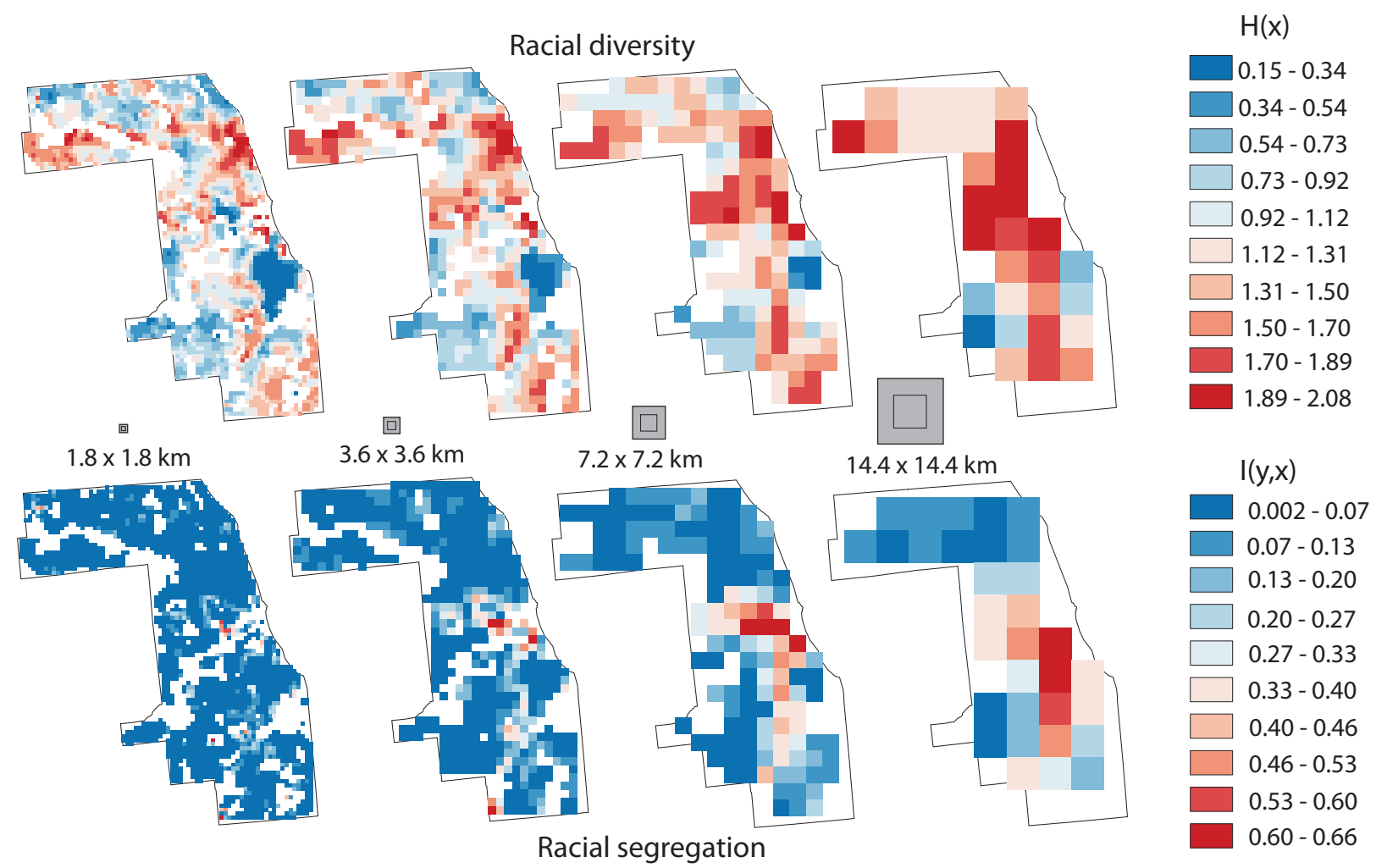

Figure 5: Spatial distribution of $H(x)$ (diversity) and $I(y, x)$ (segregation) measured at different spatial scales in the Cook County, IL in 2010. Gray squares represent the scale. The larger square is an area over which the metrics are calculated; the number below corresponds to this area. The smaller square is the size of a cell in a gridded map.

ables there is a high degree of agreement between theirs and our county-scale results. The scale $1.8 \mathrm{~km}$ roughly corresponds to the spatial scale of a typical tract in the Cook County, so we can compare Stepinski and Dmowska (2019) tract results to our results at $1.8 \mathrm{~km}$ scale. At that scale there is a good agreement with regard to diversity, but, although both methods indicate low segregation (the same segregation type, see below), the RL method yields lower values. The interesting difference between the two methods on the small scale is that one indicates a slight increase in segregation during the 1990-2010 period whereas the other indicates a slight decrease in segregation.

While Table 2 gives county-average values $H(x)$ and $I(y, x)$ at five spatial scales, Fig. 5 shows the spatial distribution of these metrics. At small scales, there are distinct areas of high and low diversity, but at large scale the majority of the Cook County is highly diverse. At the small scale, the segregation is small almost everywhere, but at a large scale, there are some highly segregated areas.
Another way to visualize the spatial distribution of racial topography is via classification of configurations of racial patterns. A configuration of racial pattern at each $L$-site is encapsulated by two metrics, $H(x)$ and $I(y, x)$ (a point on the 2-dimensional diversitysegregation diagram). On such a diagram, nearby points correspond to $L$-sites with similar racial pattern configurations. Dividing the $H-I$ diagram into a regular $3 \times 3$ grid of sectors (see the left part of Fig.6) and grouping all $L$-sites whose metrics-points are in the same sector, we obtain a mappable classification of racial patterns.

We provide an exemplar for each occurring pattern type (only seven out of nine types occur in Cook County). Note that particular choice of races present in each exemplar is irrelevant as the type refers only to configuration or the geometric character of the pattern and not to what specific races form the pattern. Moving from left to right on the grid of types the patterns are characterized by the increasing level of diversity, from racially uniform to racially diverse. Moving from bottom to top on the grid of types the patterns are charac- 

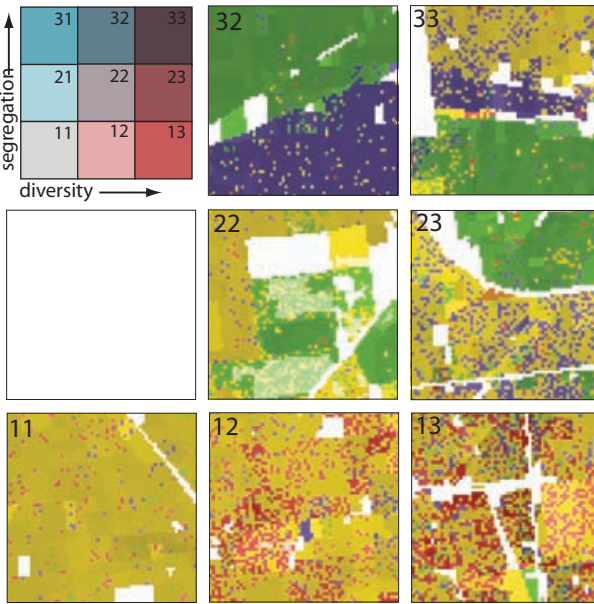
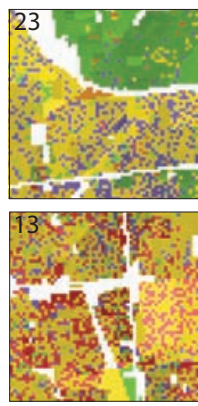
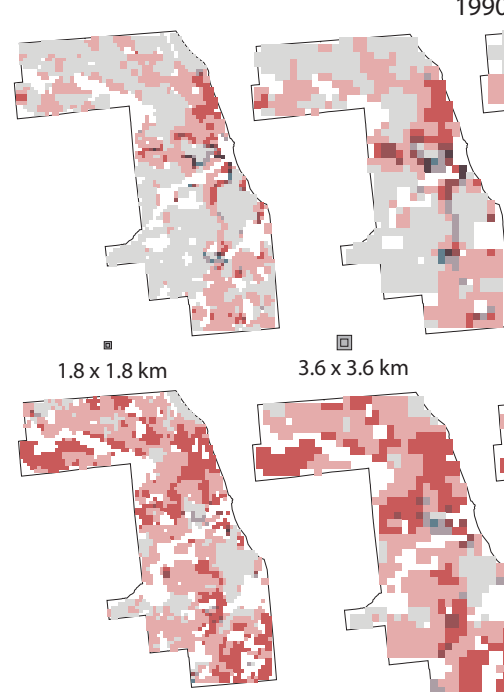

回

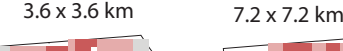

$7.2 \times 7.2 \mathrm{~km}$
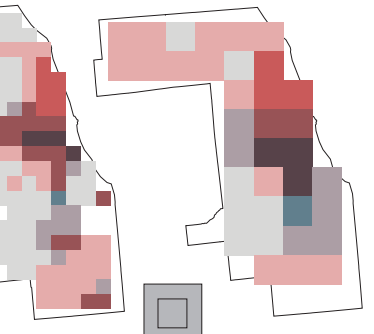

$14.4 \times 14.4 \mathrm{~km}$

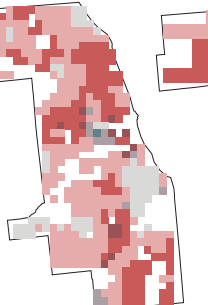

2010

Figure 6: Classification of racial topography of the Cook County into configuration types on the basis of diversity $H(x)$ and segregation $I(y, x)$. (Left) Legend racial configuration types; a configurational exemplar of each class is given. (Right) Spatial distribution of configurational classes at different scales in 1990 and 2010.

terized by the increasing level of segregation, from spatially integrated to racially segregated (see the left part of Fig.6).

A map of pattern types shows, in a single depiction, the spatial distribution of both diversity and segregation (see the right part of Fig.6). It can be constructed on multiple spatial scales and provides a convenient way to asses temporal changes in racial topography. For example, Fig. 6 shows that large parts of the Cook County in 1990 were racially uniform (and thus also integrated) on scales up to $3.6 \mathrm{~km}$ (see an exemplar for type 11 to get a sense of what that meant on the ground). However, in 2010, these parts transitioned to types 12 and 13 , which are more diverse but still not spatially segregated. Highly segregated patterns (types 32 and 33) are rare, and they are found only at large spatial scales in 1990.

\section{Conclusions and Discussion}

In this paper, we presented a novel methodology of analyzing a spatio-racial distribution of a multiracial population. What distinguishes this method is its spatial or "geographical" underpinning. As such, the method can not only quantify demographic concepts of diversity and segregation but also yields itself naturally to mapping - i.e., it visualizes a spatio-racial distribution itself as well as spatial distributions of racial diversity and segregation. On the quantitative side, to the best of our knowledge, RL is the only method that can calculate segregation without using aggregation zones thus eliminating a dilemma what scale aggregation zones to use. RL can quantify segregation for a region of arbitrary size and shape making possible to assess the average strength of segregation on multiple scales (Table 2). In addition, RL metrics of diversity and segregation are rooted in the information theory and thus have interpretations beyond their definitions (section 2.3).

On the visualization side, RL outputs maps that provide compelling visualization of spatio-racial distribution (see Fig. 2 and Fig. 4) on par with a visualization provided by the dot map technique which, however, has no quantification component. Maps of spatial variability of diversity and segregation in a given region (see Fig. 5) can be constructed by applying RL to a tessellation of a region into a grid of overlapping tiles (see Fig. 5). The spatial distribution of an overall racial character of neighborhoods can be mapped and its temporal change can be assessed (see Fig. 6).

As the 2020 US census approaches the interest in quantifying, analyzing, and communicating racial topography in US cities is once again on the rise. Indubitably, once the 2020 census race data is released, there will be a series of analyses on the state and change of racial segregation. Although the literature on how to measure and analyze racial segregation is vast (see ref- 
erences in the introduction) and currently used concepts and methods are widely accepted, we submit that Racial Landscape - the method presented in this paper - offers an attractive alternative.

The RL method approaches the problem of racial analysis from the point of view that is significantly different from the present state of the art; it does not improve the present state of the art, it moves it into a different direction. Therefore, in order to understand its logic and its explanatory power, entrenched concepts need to be set aside. Examples of such concepts include data model (racial grid vs. census tables), segregation (samerace adjacencies vs. difference in diversity between the region and its subregions), scale (measurement vs. geographical), visualization (an integral part of the method vs. not part of the method).

Once the reader accepts that the RL method cannot be understood in conventional terms, the method is conceptually simple - the spatial distribution of racial subpopulations forms a landscape which is quantified using landscape quantification methods. Computational implementation of the RL method is relatively complex, but we provide the $\mathrm{R}$ package raceland 1.0.1 (https://nowosad.github.io/raceland/) which can be used to perform the full analysis as described in sections 2 and 3 . In the remainder of this section, we discuss how different elements of the RL method differ from concepts in current methods and why, in our opinion, using the RL method offers an advantage over using current methods.

Data model. Although ultimately all methods use census data, the RL model uses dasymetrically modeled high-resolution grids in which sub-block uninhabited areas are identified. Such grids are available for the entire conterminous US for 1990, 2000, and 2010, and will be available for 2020 . The $\mathrm{R}$ implementation of the method also allows using data in the form of census blocks which are, in the first step, rasterized to the grid. However, in this case, sub-block uninhabited areas are not identified. Using a high-resolution racial grid instead of census zones has numerous advantages which are summarized in Dmowska et al. (2017).

Segregation. The RL achieves local spatial unmixing of different races so each cell is monoracial, while, at the same time, the entire region preserves its racial topography. This is an important novelty because it allows considering segregation in terms of same-race adjacencies which corresponds directly to the notion of segregation as a state of setting inhabitants of different races apart. The degree of segregation is calculated without subdividing the region, and it applies to the entire region as well as to any arbitrarily defined subregions.
In current methods, whether based on census zones (Fischer et al., 2004; Farrell, 2008; Bellman et al., 2018) or local environments (Lee et al., 2008; Östh et al., 2014), direct calculation of segregation is not possible because locations of single-race inhabitants are not explicit in the census zoned data. Instead, segregation is assessed using $\mathcal{H}$ (see section 2.3). The $\mathcal{H}$ index is a difference between the diversity of the entire region and a population-weighted average of diversities of subregions divided by the diversity of the entire region. Large values of $\mathcal{H}$ indicate that subregions are, on average, more homogeneous than the entire region - an indirect and fuzzy version of segregation. Roberto (2016) noted that the use of $\mathcal{H}$ as a measure of segregation is problematic and proposed to use the divergence index $\mathcal{D}$ instead. The divergence index can be shown to be equal to $\mathcal{H} H(x)$ or a non-normalized Theil's index. Stepinski and Dmowska (2019) noted that values of $\mathcal{H}$ are high for regions of low diversity indicating, contrary to all expectations, that such regions are "segregated." Overall, the RL method provides a more direct and robust measure of multigroup segregation than the Theil's index does.

Landscape metaphor. By using the landscape metaphor the RL is a WYSIWYG (what you see is what you get) method. This means complete integration of quantitative analysis and visualization of racial topography. The RL method makes analyzing racial topography similar to analyzing remotely sensed images - we see and comprehend the racial topography of a region before we quantify it. On the other hand, once we quantify it, we can check whether the numerical description is in line with what is observed. Such functionality is missing from the current methods, where visualization is an afterthought. A typical output of those methods is a table listing values of $\mathcal{H}$ for different metropolitan areas in different years. It is not possible to connect these values to observations.

Another advantage of the landscape metaphor is the clear concept of racial topography. There is more to the distribution of different races throughout a region than just segregation. Such distribution forms a pattern which could be understood in terms of three features: thematic content (names of races present in the pattern), composition (relative shares of different races - building blocks of the pattern), and configuration (actual spatial arrangements of the building blocks of the pattern). The RL method concentrates on the pattern's composition and configuration - the two features of the pattern which correspond to racial diversity and racial segregation. These features characterize not only the pattern of the entire region but also a pattern of any site 
within the region. In current methods, segregation value is assigned only to the entire region while subregions are only quantified by their diversities, but in the RL method, any subregion is characterized by its diversity level and its segregation level. In other words, any subregion has its own local racial landscape. These landscapes can be classified and mapped (see Fig. 6).

Multiscale analysis. In the RL method, the term scale in the phrase "multiscale analysis" refers to a measurement scale - the size of spatial sites for which racial landscapes are quantified. This allows asking the following question: What is a typical racial pattern in a subregion of size $L$ ? In the case of the Cook County, the answer to this question is given in Table 2 and Figs. 5 and 6 - racial patterns of increasingly large subregions of the Cook County are increasingly diverse and segregated. This is a direct and unambiguous application of multiscale analysis because what is analyzed are patterns in regions having different sizes.

In current papers on multiscale racial analysis, the scale refers to the size of local environments whose diversities are compared to the diversity of the entire region using the $\mathcal{H}$ index. Using different sizes of local environments results in different values of $\mathcal{H}$ (different values of segregation). Because small local environments tend to be racially homogeneous and the entire region is not, the segregation (of the entire region subdivided into small local environments) at a small scale is large. Conversely, a large local environment has diversity comparable with the entire region so the segregation (of the entire region subdivided into large local environments) at large scale is small. This is an indirect application of multiscale analysis because what is analyzed is always the same region but at different resolutions. One can indirectly infer the sought-after information that progressively larger subregions are progressively more diverse although no information can be inferred about the progression of their segregations. It also can be ambiguous because many authors tend to make a shortcut and write "segregation is higher at a finer spatial scale" (see, for example, Logan et al. (2015), which may be misunderstood as a claim that segregation of smaller subdivisions is larger.

Other. Finally, the RL data model allows for analysis that goes beyond the issues of segregation and diversity. By applying the connected component algorithm (Rosenfeld and Pfaltz, 1966) to the RL all individual racial enclaves are identified independently from the Census boundaries. This allows calculating the size distribution of enclaves (all together or for each race separately). The size distribution of single-race enclaves can be used to investigate aggregation tendencies for each racial subpopulation. The size distribution of all enclaves may provide clues to the factors driving the evolution of racial topography.

Acknowledgments. This work was supported by the University of Cincinnati Space Exploration Institute.

\section{References}

Apparicio, P., Martori, J. C., Pearson, A., Fournier, E., Apparicio, D., 2014. An open-source software for calculating indices of urban residential segregation. Social Science Computer Review 32(1), $117-128$.

Ard, K., 2016. By all measures: An examination of the relationship between segregation and health risk from air pollution. Population and Environment 38(1), 1-20.

Bailey, N., Barnes, H., Livingston, M., Mclennan, D., 2012. Understanding neighbourhood population dynamics for neighbourhood effects research: a review of recent evidence and data source developments. In: In Understanding neighbourhood dynamics. Springer, Dordrecht, pp. 23-41.

Bellman, B., Spielman, S. E., S., R., Franklin, 2018. Local Population Change and Variations in Racial Integration in the United States, 2000-2010. International regional science review 41(2), 233-255.

Bråmå, A., 2008. Dynamics of ethnic residential segregation in Göteborg, Sweden. Population, Space and Place 14(2), 101-117.

Clark, W. A., Anderson, E., Östh, J., Malmberg, B., 2015. A multiscalar analysis of neighborhood composition in Los Angeles, 2000-2010: A location-based approach to segregation and diversity. Annals of the Association of American Geographers 105(6), 1260-1284.

Dmowska, A., Stepinski, T. F., 2019. Racial Dot Maps Based on Dasymetrically Modeled Gridded Population Data. Social Sciences 8(5), 157.

Dmowska, A., Stepinski, T. F., Netzel, P., 2017. Comprehensive framework for visualizing and analyzing spatio-temporal dynamics of racial diversity in the entire United States. PLoS ONE 12(3), e0174993.

Farrell, C. R., 2008. Bifurcation, fragmentation or integration? The racial and geographical structure of US metropolitan segregation, 1990-2000. Urban Studies 45, 467-499.

Farrell, C. R., Lee, B. A., 2011. Racial diversity and change in metropolitan neighborhoods. Social Science Research 40 (4), 1108-1123.

Finney, N., 2012. How ethnic mix changes: Typologising neighbourhood population dynamics of ethnic groups. In: Understanding Neighbourhood Dynamics. Springer, Dordrecht, pp. 203-224.

Fischer, C. S., Stockmayer, G., Stiles, J., Hout, M., 2004. Distinguishing the geographic levels and social dimensions of US metropolitan segregation, 1960-2000. Demography 41(1), 37-59.

Fowler, C. S., 2016. Segregation as a multiscalar phenomenon and its implications for neighborhood-scale research: The case of South Seattle 1990-2010. Urban Geography 37(1), 1-25.

Frank, S. A., 2009. The common patterns of nature. Journal of evolutionary biology 22(8), 1563-1585.

Gergel, S. E., Turner, M. G., 2017. Learning landscape ecology: a practical guide to concepts and techniques. Springer.

Giordano, A., Cheever, L., 2010. Using dasymetric mapping to identify communities at risk from hazardous waste generation in San Antonio, Texas. Urban Geography 31(5), 623-647.

Haralick, R. M., Shanmugam, K., Dinstein, I., 1973. Textural features for image classification. IEEE Transactions on systems, man, and cybernetics 6, 610-621. 
Hennerdal, P., Nielsen, M. M., 2017. A multiscalar approach for identifying clusters and segregation patterns that avoids the modifiable areal unit problem. Annals of the American Association of Geographers 107(3), 555-574.

Hong, S. Y., O’Sullivan, D., Sadahiro, Y., 2014. Implementing spatial segregation measures in R. PlosS one 9(1), e113767.

Iceland, J., 2004. Beyond Black and White metropolitan residential segregation in multi-ethnic America. Social Science Research 33, 248-271.

Johnston, R., Poulsen, M., Forrest, J., 2007. Ethnic and racial segregation in U.S. metropolitan areas, 1980-2000: The dimension of segregation revisited. Urban Affairs Review 42 (4), 479-504.

Jones, K., Johnston, R., Manley, D., Owen, D., Charlton, C., 2015. Ethnic residential segregation: A multilevel, multigroup, multiscale approach exemplified by London in 2011. Demography 52(6), 1995-2019.

Lee, B. A., Reardon, S. F., Firebaugh, G., Farrell, C. F., Matthews, S. A., O'Sullivan, D., 2008. Beyond the Census Tract: Pattern and Determinants of Racial Segregation at Multiple Geographic Scales. American Sociological Review 73 (5), 766-791.

Logan, J. R., Zhang, W., Chunyu, M. D., 2015. Emergent ghettoes: Black neighborhoods in New York and Chicago, 18001940. American Journal of Sociology 120, 1055-1094.

Louf, R., Barthelemy, M., 2016. Patterns of residential segregation. PloS one 11(6), e0157476.

Maantay, J., Maroko, A., 2009. Mapping urban risk: Flood hazards, race, \& environmental justice in New York. Applied Geography 29(1), 111-124.

Massey, D. S., Denton, N. A., 1988. The dimensions of residential segregation. Social forces 67(2), 281-315.

Nowosad, J., Stepinski, T. F., 2018. Global inventory of landscape patterns and latent variables of landscape spatial configuration. Ecological Indicators 89, 159-167.

Nowosad, J., Stepinski, T. F., 2019. Information Theory as a consistent framework for quantification and classification of landscape patterns. Landscape Ecology 34(9), 2091-2101.

Olteanu, M., Randon-Furling, J., Clark, W. A., 2019. Segregation through the multiscalar lens. Proc. Natl. Acad. Sci. 116, 1225012254.

Openshaw, S., 1984. The modifiable areal unit problem. Vol. 38. CATMOG (Concepts and Techniques in Modern Geography), Norwich: Geobooks.

Östh, J., Clark, W. A. V., Malmberg, B., 2014. Measuring the scale of segregation using k-nearest neighbor aggregates. Geographical Analysis 47 (1), 34-49.

O'Sullivan, D., Wong, D. W., 2007. A Surface-Based Approach to Measuring Spatial Segregation. Geographical Analysis 39(2), 147-168.

Reardon, S. F., Firebaugh, G., 2002. Measures of Multigroup Segregation. Sociological Methodology 32(1), 33-67.

Reardon, S. F., Matthews, S. A., Sullivan, D. O., Lee, B. A., Firebaugh, G., Farrell, C. R., 2008. The geographic scale of metropolitan racial segregation. Demography 45 (3), 489-514.

Reardon, S. F., Sullivan, D. O., 2004. Measures of spatial segregation Sociological methodology 34, 121-162.

Roberto, E., 2016. The Divergence Index: A Decomposable Measure of Segregation and Inequality. arXiv preprint, 1508.01167.

Rosenfeld, A., Pfaltz, J. L., 1966. Sequential operations in digital picture processing. J. ACM 13(4), 471-494.

Roth, R. E., 2010. Dot Density Maps. In: Warf, B. (Ed.), The Encyclopedia of Geography. Thousand Oaks, CA, Sage, pp. 787-790.

Sadahiro, Y., Hong, S. Y., 2014. Decomposition approach to the measurement of spatial segregation. Tech. rep., CSIS Discussion Paper No.119R.

Shannon, C. E., 1948. A mathematical theory of communication. Bell system technical journal 27(3), 379-423

Shertzer, A., Walsh, R. P., 2019. Racial sorting and the emergence of segregation in American cities. The Review of Economics and Statistics 101(3), 1-14.

Stepinski, T. F., Dmowska, A., 2019. Imperfect melting potAnalysis of changes in diversity and segregation of US urban census tracts in the period of 19902010. Computers, Environment and Urban Systems 76, 101-109.

Theil, H., Finizza, A. J., 1971. A Note on the Measurement of Racial Integration of Schools by Means of Informational Concepts. The Journal of Mathematical Sociology 1(2), 187-193.

Wong, D., 2004. Comparing Traditional and Spatial Segregation Measures: A Spatial Scale Perspective. Urban Geography 25, 66-82.

Wong, D. W., 2016. From Aspatial to Spatial, from Global to Local and Individual: Are We on the Right Track to Spatialize Segregation Measures? In: Howell, F. M., Porter, J. R., Matthews, S. A. (Eds.), Recapturing Space: New Middle-Range Theory in Spatial Demography. Springer International Publishing, pp. 77-98.

Yao, J., Wong, D. W., Bailey, N., Minton, J., 2018. Spatial segregation measures: A methodological review. Tijdschrift voor economische en sociale geografie 110(3), 235-250.

Yizhaq, H., Portnov, B., Meron, E., 2004. A mathematical model of segregation patterns in residential neighbourhoods. Environment and Planning A 36(1), 149-172. 